\title{
Assessing the Efficiency of a Proposed Project in Waste Management
}

\author{
Scorțar Lucia-Monica \\ Babeş-Bolyai University, Cluj-Napoca \\ Romania
}

\section{Introduction}

This paper addresses a current and very important problem, namely household waste generation that, on the one hand, affects the environment and human health and on the other hand, it reflects how inefficient the usage of natural resources by the society is.

Nature is a good human environment, more or less altered by people, and mankind is part of it. Environment means all the elements that surround us, humans often leaving their footprint on them, mostly in a destructive way; hence the need of interventions from the state authorities for nature protection and conservation. Leniently watching people's actions that destroy nature and the environment, would mean a distortion of their own sense of conservation, a degradation of personal and community life.

If industrialization and urbanization are activities more than necessary in human social development, humans also have to find alternatives to the process of altering the environment, in order to preserve a clean environment so that life can exist and be as more beautiful.

It can be noted by everyone that even where a single human lives, he makes some changes to the environment and, implicitly, creates waste, especially household waste. The larger the community, the bigger the waste quantity is, so that the existence of uncontrolled, accumulated household creates major problems that must be solved urgently and permanently. As a result, they must be managed so as not to burden to suffocation the community, thus imposing waste selective collection, reuse, recycling and treatment, and finally storing waste remains.

In the current waste management strategies, the trend is an integrated system based on prevention of waste, minimizing waste quantity, recycling and reusing of waste, treatment with a large number of technology, and ultimately, waste remains disposal, also taking care of population and environment health ( "waste management hierarchy").

At the beginning of XXI century, we believe that not us, but generally people do not have the required education to treat waste problem as something serious that can influence decisively our future existence.Technical creativity in waste recovery did not have the same pace as the creativity in developing new products and adopting and implementing the technologies for achieving them. We believe that, viewed as an actual and future businesses, waste recovery will find its required technical capacity. 
Once the waste was produced, the best way to reduce or eliminate its negative impact on the environment is its recovery. Recovering household waste fractions requires a separation between recoverable components. Since the separation of mixed waste is already done at great expense and often with insufficient results, useful materials must be collected by collection systems and recovered before mixing them with other parts of the waste.

This paper is proposing a new investment project in household waste management for a group of associated communities in both urban and rural areas, forming Comunitatea Urbană Arieş, județul Cluj (CUA).

Comunitatea Urbană Arieş is an association of local authorities from the area Arieş-TurdaCâmpia Turzii with public institution vocation and attributions for cooperation created through the free will act expressed by the participating Local Councils, according to their legal prerogatives, with the current regulations and the regulations of the European Union. It is appointed by the member councils to perform for them and on their behalf public services of common interest.

The fundamental criterion for joining and participating in the Comunitatea Urbană Arieş is for each Local Council the efficient access to common resources and their rational and integrated management, in order to protect the environment (Comunitatea Urbană Arieş, Statutul Asociației, Turda, 2005).

The proposed waste management scheme is analyzed based on the principle of population access to sanitation services, under which public authorities are responsible for organizing public services of the community so that all members have equal access to these services.

The analysis showed that the effectiveness of the appropriate scheme for this area cannot be conceived outside a recovery circuit for useful material contained in household waste (paper/cardboard, glass, metal, plastics, including the capitalization of organic waste).

This project demonstrates a sustainable approach to waste management, proposing investments in the purchase of containers for selective collection, transport facilities, sorting, composting, recycling, organic waste incineration and final ecological disposal.

This project is designed to serve Comunitatea Urbană Arieş (117.780 inhabitants and 2.975 businesses) which is composed of two urban (Turda and Câmpia Turzii) and nine surrounding rural areas (Mihai Viteazu, Călăraşi, Frata, Aiton, Luna, Petreştii de Jos, Sănduleşti, Tritenii de Jos, Viişoara).

We believe that the modern system of sustainable management of waste proposed for Comunitatea Urbană Arieş will contribute to reducing the amount of waste stored in this area, as this is a suitable system which treats the main fraction of household waste to protect the environment.

\section{Goal, targets, location}

Waste management situation in the studied area cannot be extended for too long, as uncontrolled waste accumulations are affecting both environmental health and that of the population. The proposed investment project includes activities related to the hierarchy in terms of waste management (prevention, collection, recovery and disposal), as there is no proper system of waste management in this area. 
This project concerns both actions in collection, transport, recycling and composting useful materials of household waste and a further step in remaining waste incineration, in an incinerator equipped with two modules. Thus, only the ashes resulting from incineration are finally stored.

These actions that should be provided in an integrated waste management system are phased as follows:

1. Waste processing at the source (in the apartment, household, institution),

2. Providing selective waste collection service (collection container, where the public service responsibility begins),

3. Waste transport with special vehicles,

4. Interim storage of waste (transfer station located between Câmpia-Turzii and Turda),

5. Recovery of selected waste fractions will be done by special institutions, and mixed collected waste will be treated in the sort-treatment station located on the former chemical plant Turda platform

6. Operating a waste incineration plant for the unused remaining waste from the sorting station located between Turda and Câmpia-Turzii

7. Transporting of the resulting ash to the ecological county landfill that will be opened in the village Feleacu.

In this scheme, the sorted waste will be recovered through their direct sale to recyclers. In this way, incomes can be obtained that will allow support of operating expenses with direct impact for the sanitation tariffs. Based on this scenario, we will forecast costs and revenues involved in the functioning of this waste management scheme proposed in Comunitatea Urbană Arieş and we will determine the efficiency of this system.

Applying this investment project is aimed at: empowering people in terms of practical activities for the selective collection of waste, increasing the coverage of sanitation services at bearable price, reducing quantities of waste and recovery of useful materials contained in waste, the final goal of these actions being a healthy and clean environment.

a. Non-selective collection of waste for the envisaged project consists of:

- For the urban areas: Collecting waste from households includes: systematic structure of the current collection routes, extending these routes and have them served by the sanitation department, which will be reorganized in accordance with local laws and regulations of sanitation. Waste collection service will be extended.

The operating principle is collection at the collection points, respectively, each household will have pre-collection containers and collection points will be grouped as follows: in block of flats areas collection points will be arranged for each 200 apartments, and in houses areas the collection vehicles will travel from door to door to collect the waste.

- For the rural areas: Collection and transport vehicles will retrieve mixed waste from door to door, covering the rural area. Social and administrative areas (administrative units, commercial units, schools and kindergartens) will benefit of the purchase of a number of pre-collection containers with a capacity of $1,1 \mathrm{~m}^{3}$, used for urban areas only.

b. Selective collection of recoverable waste consists of: 
- For the urban areas: In order to selectively collect waste, we propose organizing selective waste collection points, each having four containers for recoverable waste. We propose the collection of four fractions of recoverable waste: glass (in a 2compartment container), metal, paper/cardboard and plastic. In houses areas, for every 100 households there will be arranged a point of collection, and in blocks of flats areas, for each 200 apartments, a collection point will be arranged.

- For the rural areas: For the selective waste collection, collection points will be arranged in each village. Selective collection points in villages are designed to serve 150 rural households, because of the lower waste ratio generated as opposed to the urban areas. Collection points structure is identical to those in urban areas.

\section{Interpretation and analysis data}

The poroposed waste management scheme is sized for a service area that includes 117.780 people and 2.975 companies, dispersed in two urban and nine rural areas, forming Comunitatea Urbană Arieş. (Studiu de Oportunitate privind delegarea gestiunii serviciului de salubritate în municipiul Turda, GPA Business Consulting, August 2006).

After the analysis and the description of the proposed waste management scheme, the object estimates of the investment components have been set and also a general estimate of the proposed management scheme has been developed, as follows:

\begin{tabular}{|c|l|c|}
\hline Object estimate & \multicolumn{1}{|c|}{ Investment components } & $\begin{array}{c}\text { Value of the objects } \\
\text { estimate (with VAT) }\end{array}$ \\
\hline Object estimate 1 & Setting up 388 collection points & 3.069 .386 euro \\
\hline Object estimate 2 & Purchasing transportation means & 773.500 euro \\
\hline Object estimate 3 & Setting up the transfer station & 450.466 euro \\
\hline Object estimate 4 & Setting up the sorting/treating station & 4.838 .724 euro \\
\hline Object estimate 5 & $\begin{array}{l}\text { Purchasing and distributing collection } \\
\text { containers to the population }\end{array}$ & 555.603 euro \\
\hline Object estimate 6 & Setting up the incineration station & 4.941 .957 euro \\
\hline
\end{tabular}

Tabel 1. The object estimates of the investment components

The general total sum of the investment including VAT is situated at the value of 14.629 .636 Euro. This value, although it seems a high value, compared to the usual schemes promoted in our country cover those segments of the management scheme which are not usually included in the budgets of the projects which need financing, such as the distribution of collection containers to the population, including the rural area, as well as the expenditures for setting up a modern sorting/treating station.

Determining the precision of the total investment value depends on both information sources and how to deepen the calculations during the project's execution design. The value of the object estimate is obtained by summing the values of the works categories that make the object. The value of these works categories is estimated on the base of the works quantities and their prices. 
In order to achieve this investment objective, after drawing the general estimate, the total investment value of 14.629 .636 euro resulted, obtaining the following values for categories of work:

- Expenses for obtaining the land: they are considered 0, because the land on which the investment objectives are placed belong to the public domain and will be made available to the project by the beneficiary City Council;

- Expenses for land planning (enbankments): 2.542 .000 euro;

- Expenses for utilities necessary for the objective (wiring, plumbing, heating, telecommunications equipment): 73.640 euro;

- $\quad$ Expenses for design and technical assistance: 12.980 euro;

- Expenses for basic investment (construction and installation, equipment, assembling the machineries): 11.484 .672 euro;

- $\quad$ Other expenses (site organization, fees, taxes, extraordinary expenses): 178.320 euro;

- Expenses for technological tests and delivering to the beneficiary (including training of operating personnel): 53.690 euro.

Household waste generated by Comunitatea Urbană Arieş, in 2009, was 45.417 tons distributed as follows:

- In the urban environment there have been generated 27.170 tons;

- In the rural areas there have been generated 5.127 tons;

- $\quad$ The amount generated by businesses was 13.127 tons. Based on these data from local authorities and taking into account the waste generation index for urban and rural areas (according to County Plan for Waste Management), a forecast has been done for the quantities of household waste generated in this area. Generation indicators are calculated both for municipal waste and for household waste, based on the generated quantity and on the number of persons served. Projected quantities of recoverable materials (paper, glass, metals, plastics) from individuals and businesses in the Comunitatea Urbană Arieş were determined by multiplying the amounts recovered by the number of individuals and businesses served.

The quantity of household waste treated in the treatment station is calculated as the difference between household waste generated and waste recovered. Biodegradable waste occupies a large proportion of the total amount of household waste generated by the studied population, namely:

- $\quad 61 \%$ biodegradable waste is generated in urban areas;

- $\quad 55 \%$ biodegradable waste is generated in rural areas.

This waste fraction will be treated in the sort-treatment station, the compost beeing properly capitalized. Final waste is waste that remains after the fraction of biodegradable waste was recovered from household waste for treatment. This fraction will be stored safely on the green ramp Feleacu.

The water content of biodegradable waste to be processed is considered $30 \%$. A $70 \%$ of dry matter is recovered as compost used for agricultural areas and the remaining $30 \%$ is considered final residue being deposited. 
Next, we consider as useful the forecast of the total amount of household waste deposited at the landfill, by aggregating the quantities of waste that remains after treatment (waste treatment) and the amount of residue resulting from the composting process.

Analysis horizon of the proposed project for Comunitatea Urbană Arieş is 21 years, the recommended duration for waste management projects being between 20 to 30 years. The analysis horizon of the project (economic lifetime) is the time for which it is expected to obtain the project's benefits.

Waste incineration involves burning waste in special facilities called incinerators, which ensure high combustion temperatures that determine the neutralization of waste using containment and gas purification equipments.

The majority of modern solid waste incinerators produce less particulate and gaseous pollutants than their predecessors, which had few environmental controls on air emissions by regulatory bodies world-wide, multi stage pollution control systems are becoming more common. The operation of the combustion process plays an important role in the formation of some pollutants. Carbon monoxide, nitrogen oxides, hydrocarbons and other volatile organic compound emissions can be minimised by optimising the combustion process. The combustion of waste can produce trace quanties of dioxins and furans.( McDougall et al., 2001)

Reintroducing the useful materials contained in household waste in the manufacturing process yields clear advantages. Recovered materials have been previously purified and processed so that their use in manufacturing activity involves a cleaner environment and less energy consumption.

As a result of waste incineration, only $20 \%$ of the input incineration waste will be landfilled, in the form of unrecoverable slag and ash, this deposited refuse requiring storage expenses provided in the following table. The following expenses are required for processing the waste in the incineration plant:

- Expenditure on fuel for the flame holder: 60 euros per ton of waste burned;

- Environmental monitoring expenses: approx. 75.000 euros annually;

- Maintenance and operating costs (salaries, overhead, materials, maintenance, repairs)

Starting with 2013, vehicles and equipment maintenance is estimated at 168.000 euro / year due to higher degree of wear, while in the first four years it is estimated that the maintenance will be about half, i.e. 84.000 euro.

Implementing this project will create 67 jobs on indefinite term (stations heads, electromechanical maintenance workers, administrative staff, drivers, etc..) and expected salary level for the first year of operation is 501.840 euro.

- Used waters purification expenditure: about 1,2 euros per ton of processed waste;

- Gas cleaning costs: replacing filter cartridges, about 13 euros per ton of processed waste;

- Expenditure with environmental taxes: about 5 euros per ton of processed waste (environment fund contributions, local eco-taxes, stock registration system of green certificates for $\mathrm{CO} 2$ emissions, eco-audit costs etc.).

Depreciation expenses (investment value/number of years of operation): 247.098 euro. 


\begin{tabular}{|c|c|c|c|c|c|c|c|c|c|c|c|c|c|c|c|c|c|c|c|c|c|}
\hline 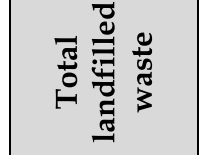 & 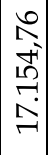 & $\begin{array}{l}0 \\
\tilde{a} \\
\sigma \\
\vdots \\
0 \\
-1\end{array}$ & 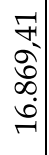 & 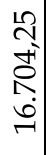 & 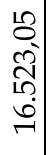 & 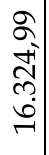 & 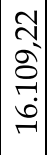 & \begin{tabular}{l}
$\infty$ \\
\multirow{N}{N}{} \\
$\infty$ \\
10 \\
10
\end{tabular} & $\begin{array}{l}0 \\
\infty \\
0 \\
0 \\
0 \\
10 \\
10\end{array}$ & 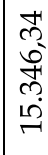 & $\begin{array}{l}\text { ิㅗ } \\
0 \\
0 \\
0 \\
10 \\
1\end{array}$ & 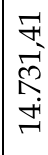 & $\mid \begin{array}{l}\infty \\
0 \\
\infty \\
\infty \\
\infty \\
+ \\
+ \\
-1\end{array}$ & 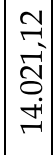 & 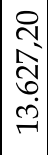 & $\mid \begin{array}{l}0 \\
0 \\
10 \\
0 \\
0 \\
0 \\
\end{array}$ & 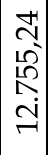 & 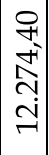 & 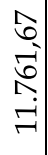 & 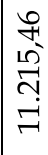 & 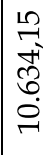 \\
\hline 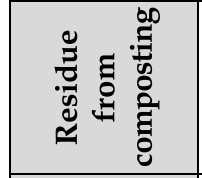 & $\begin{array}{l}0 \\
10 \\
0 \\
10 \\
\infty \\
\dot{n}\end{array}$ & $\begin{array}{l}10 \\
0 \\
0 \\
0 \\
0 \\
\dot{0}\end{array}$ & 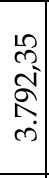 & $\begin{array}{l}\text { N } \\
\stackrel{2}{n} \\
\\
m\end{array}$ & 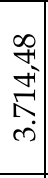 & 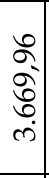 & 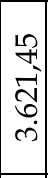 & 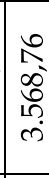 & 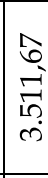 & 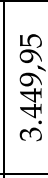 & $\begin{array}{l}\infty \\
m \\
\infty \\
\infty \\
m \\
m\end{array}$ & 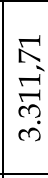 & 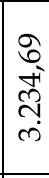 & $\begin{array}{c}5 \\
0 \\
i \\
0 \\
\\
0\end{array}$ & $\left|\begin{array}{c}0 \\
0 \\
0 \\
0 \\
0 \\
0\end{array}\right|$ & $\left|\begin{array}{l}N \\
N \\
o \\
o \\
o \\
i\end{array}\right|$ & 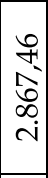 & $\begin{array}{l}0 \\
n \\
o \\
1 \\
\\
i\end{array}$ & 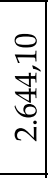 & $\begin{array}{l}\bar{n} \\
\stackrel{-}{N} \\
\text { in }\end{array}$ & 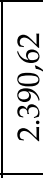 \\
\hline 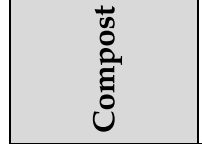 & 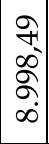 & 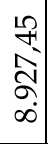 & $\begin{array}{l}-1 \\
\infty \\
\infty \\
\infty \\
\infty \\
\infty\end{array}$ & 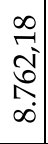 & $\begin{array}{l}\stackrel{n}{7} \\
\hat{\sigma} \\
\infty \\
\infty\end{array}$ & 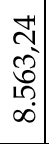 & $\begin{array}{c}0 \\
0 \\
0 \\
0 \\
10 \\
+ \\
\infty\end{array}$ & $\begin{array}{l}\text { 공 } \\
\text { Nิ } \\
\infty\end{array}$ & 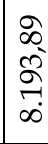 & 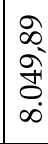 & 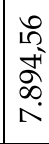 & 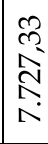 & 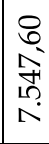 & 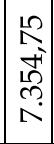 & 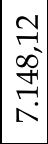 & 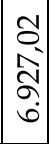 & 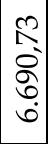 & 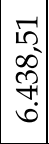 & 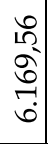 & $\begin{array}{l}10 \\
0 \\
\infty \\
\infty \\
\infty \\
10\end{array}$ & 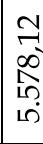 \\
\hline 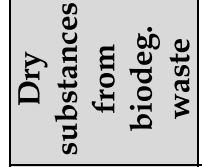 & 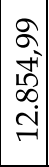 & $\begin{array}{l}\sqrt{n} \\
0 \\
10 \\
\\
\end{array}$ & 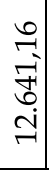 & 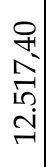 & 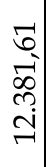 & 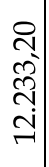 & 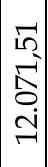 & $\begin{array}{l}\infty \\
\infty \\
12 \\
\infty \\
\infty \\
\\
1\end{array}$ & 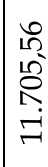 & मे & 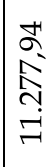 & 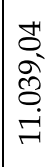 & 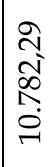 & 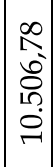 & 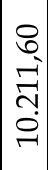 & $\begin{array}{l}+1 \\
1 \\
2 \\
\infty \\
\infty \\
\sigma\end{array}$ & $\begin{array}{c}2 \\
2 \\
0 \\
10 \\
10 \\
\sigma\end{array} \mid$ & 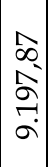 & $\begin{array}{l}12 \\
0 \\
n \\
\infty \\
\infty \\
\infty\end{array}$ & 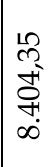 & 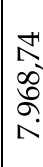 \\
\hline 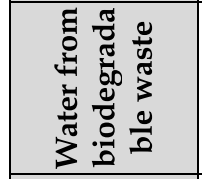 & 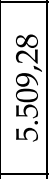 & $\begin{array}{c}2 \\
10 \\
10 \\
1 \\
10\end{array}$ & 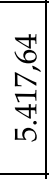 & 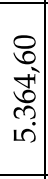 & 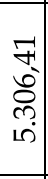 & 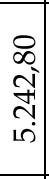 & 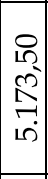 & 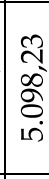 & $\begin{array}{c}0 \\
0 \\
0 \\
0 \\
0 \\
10\end{array}$ & 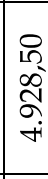 & $\begin{array}{l}\text { 움 } \\
\infty \\
2 \\
\infty \\
+\end{array}$ & 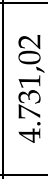 & $\begin{array}{l}\infty \\
2 \\
0 \\
\text { ర్ } \\
+\end{array}$ & $\begin{array}{c}\sigma \\
\sigma \\
\tilde{S} \\
1 \\
+i\end{array}$ & 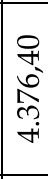 & 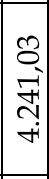 & $\begin{array}{l}0 \\
\hat{2} \\
0 \\
\delta \\
0 \\
+\end{array}$ & 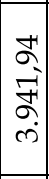 & $\begin{array}{l}\stackrel{0}{N} \\
\hat{N} \\
\hat{N} \\
\dot{m}\end{array}$ & $\begin{array}{l}0 \\
\infty \\
-1 \\
\delta \\
\dot{0}\end{array}$ & 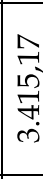 \\
\hline 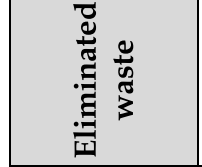 & 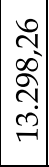 & 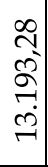 & 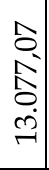 & 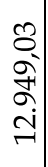 & $\begin{array}{l}1 \\
10 \\
\infty \\
\infty \\
0 \\
\text { î. }\end{array}$ & 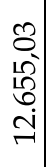 & 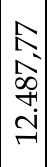 & 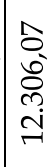 & 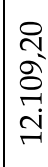 & 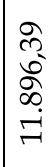 & 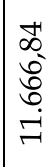 & 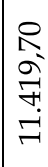 & 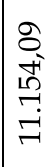 & $\begin{array}{l}8 \\
0 \\
0 \\
0 \\
0 \\
0 \\
0\end{array}$ & 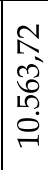 & 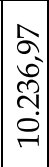 & $\begin{array}{l}\infty \\
1 \\
1 \\
\infty \\
\infty \\
0\end{array} \mid$ & 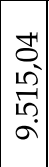 & 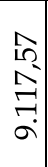 & 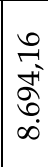 & 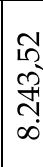 \\
\hline 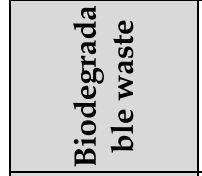 & 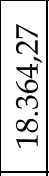 & 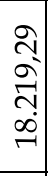 & $\begin{array}{c}0 \\
\infty \\
\infty \\
0 \\
0 \\
\infty \\
\infty \\
\\
1\end{array}$ & 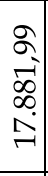 & 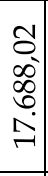 & 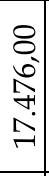 & 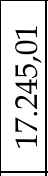 & $\begin{array}{l}\text { oे } \\
+ \\
\text { gे } \\
\text { فे }\end{array}$ & 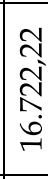 & 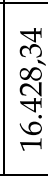 & 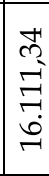 & 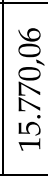 & 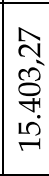 & $\begin{array}{l}9 \\
0 \\
8 \\
8 \\
10 \\
10\end{array}$ & $\begin{array}{l}\text { న్ } \\
\infty \\
\infty \\
1 \\
+\end{array}$ & 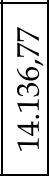 & 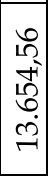 & 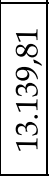 & 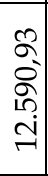 & 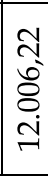 & 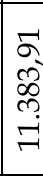 \\
\hline 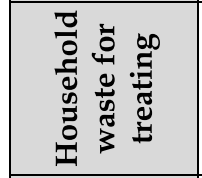 & 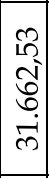 & 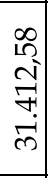 & $\begin{array}{c}\infty \\
\infty \\
1 \\
m \\
\sim \\
\stackrel{m}{-}\end{array}$ & 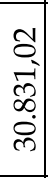 & $\begin{array}{l}\infty \\
1 \\
6 \\
o \\
+ \\
0 \\
0 \\
0\end{array}$ & 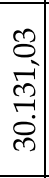 & 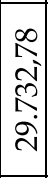 & 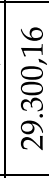 & 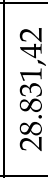 & 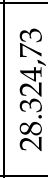 & 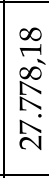 & 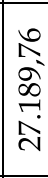 & 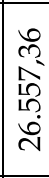 & $\begin{array}{l}\infty \\
0 \\
\infty \\
\infty \\
\infty \\
10 \\
1\end{array}$ & $\begin{array}{l}\overrightarrow{1} \\
\sqrt{n} \\
\stackrel{2}{2} \\
\stackrel{2}{2}\end{array}$ & 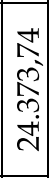 & 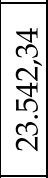 & 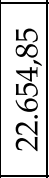 & 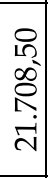 & 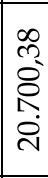 & \begin{tabular}{l}
\multirow{+}{*}{} \\
స్ \\
बे
\end{tabular} \\
\hline 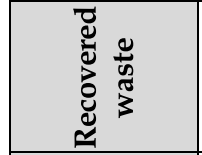 & \begin{tabular}{l}
$\Omega$ \\
\multirow{2}{n}{} \\
$\stackrel{n}{\rightleftharpoons}$
\end{tabular} & 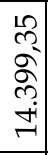 & 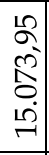 & 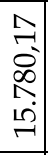 & 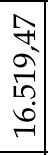 & 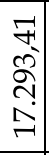 & 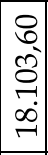 & 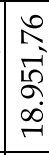 & $\begin{array}{l}10 \\
9 \\
2 \\
\infty \\
0 \\
\end{array}$ & 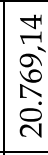 & 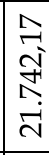 & 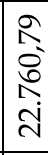 & $\begin{array}{l}\text { ?. } \\
\text { N1} \\
\infty \\
\text { ते }\end{array}$ & 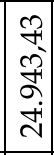 & 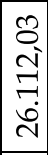 & \begin{tabular}{|l|}
$\infty$ \\
3 \\
10 \\
2 \\
2 \\
\\
\end{tabular} & 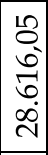 & 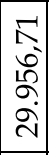 & 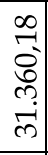 & 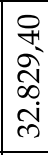 & 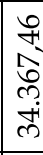 \\
\hline 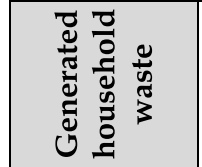 & 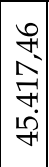 & 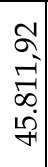 & 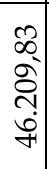 & 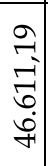 & $\begin{array}{l}\qquad 0 \\
0 \\
6 \\
0 \\
\vdots \\
\dot{y}\end{array}$ & 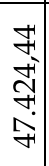 & 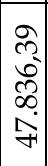 & 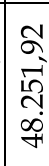 & 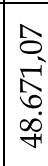 & 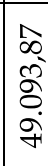 & 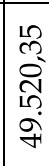 & $\begin{array}{l}10 \\
12 \\
0 \\
\alpha \\
\alpha \\
\text { q }\end{array}$ & 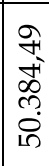 & 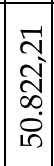 & 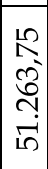 & 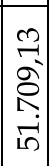 & 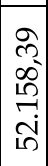 & 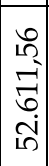 & $\begin{array}{c}\infty \\
0 \\
0 \\
0 \\
0 \\
0 \\
10 \\
10\end{array}$ & 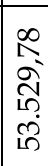 & $\begin{array}{l}8 \\
\text { ஓं } \\
\text { ळे } \\
\text { ம் }\end{array}$ \\
\hline ‡્ર & 옹 & 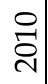 & 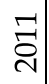 & 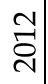 & $\stackrel{m}{\stackrel{m}{v}}$ & $\begin{array}{l}+ \\
\stackrel{d}{v}\end{array}$ & $\sim$ & 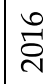 & 규 & $\stackrel{\infty}{\tilde{\sigma}}$ & 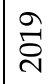 & ڤ్ & స్ర & స్ & ڤి & 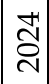 & $\begin{array}{l}\stackrel{1}{2} \\
\stackrel{్}{\mathcal{N}}\end{array}$ & 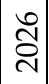 & సิ & 文 & \\
\hline
\end{tabular}

Table 2. Prediction of the waste quatities (ton) 


\begin{tabular}{|c|c|c|c|c|c|c|c|c|c|c|c|c|c|c|c|c|c|c|c|c|c|}
\hline 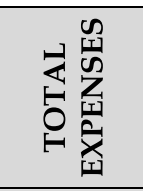 & 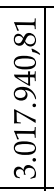 & $\begin{array}{l}50 \\
0 \\
10 \\
0 \\
\alpha \\
10 \\
0 \\
0 \\
\dot{c} \\
\end{array}$ & 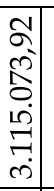 & 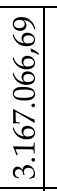 & 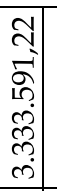 & 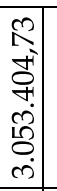 & 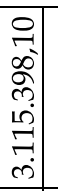 & $\begin{array}{l}\infty \\
0 \\
\infty \\
\infty \\
\sigma \\
\vdots \\
\infty \\
\dot{\infty} \\
\infty\end{array}$ & 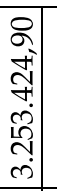 & 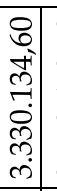 & 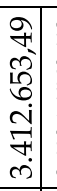 & 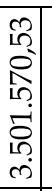 & $\begin{array}{l}8 \\
\infty \\
0 \\
0 \\
10 \\
\hat{0} \\
10 \\
\text { ஸे }\end{array}$ & 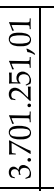 & $\begin{array}{l}0 \\
10 \\
10 \\
10 \\
10 \\
0 \\
0 \\
0 \\
0 \\
0\end{array}$ & 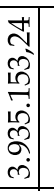 & 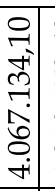 & 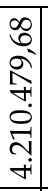 & 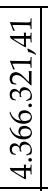 & 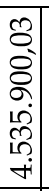 & 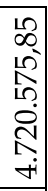 \\
\hline 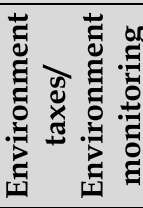 & 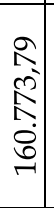 & 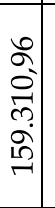 & 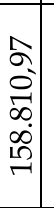 & 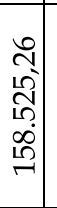 & 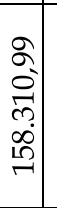 & 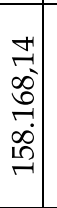 & 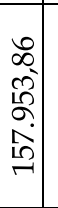 & $\begin{array}{l}0 \\
\infty \\
\hat{2} \\
\stackrel{2}{2} \\
\hat{\omega} \\
\end{array}$ & 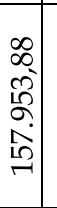 & 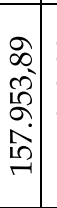 & 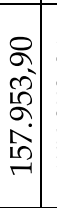 & 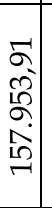 & 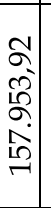 & 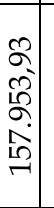 & 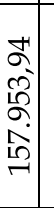 & 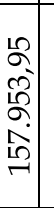 & 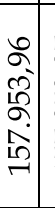 & 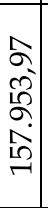 & 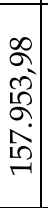 & $\begin{array}{l}\text { જે } \\
\text { مి } \\
\text { ૂે } \\
\hat{1}\end{array}$ & 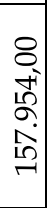 \\
\hline 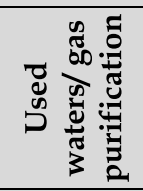 & 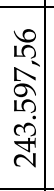 & 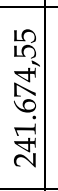 & 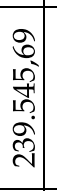 & 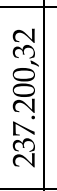 & 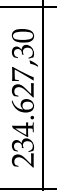 & 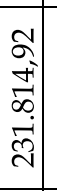 & 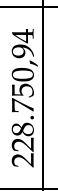 & 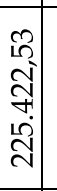 & 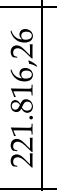 & $\begin{array}{l}0 \\
\infty \\
\infty \\
\sigma \\
\\
\vec{\nu}\end{array}$ & 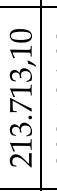 & 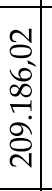 & 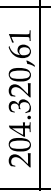 & $\begin{array}{l}\Omega \\
\sigma \\
\delta \\
\delta \\
\stackrel{2}{\sigma}\end{array}$ & 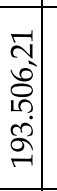 & 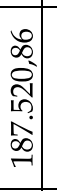 & 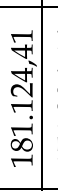 & 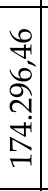 & 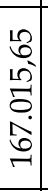 & 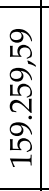 & 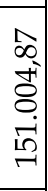 \\
\hline 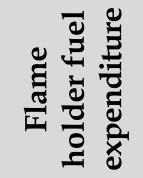 & 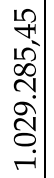 & $\begin{array}{c}0 \\
8 \\
0 \\
0 \\
ت \\
\overrightarrow{-} \\
0 \\
-\end{array}$ & 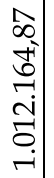 & 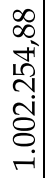 & 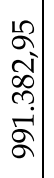 & 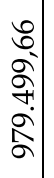 & 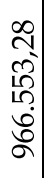 & 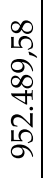 & 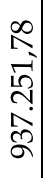 & 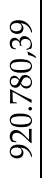 & $\begin{array}{c}0 \\
0 \\
0 \\
0 \\
\vdots \\
\dot{0} \\
\varnothing\end{array}$ & 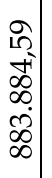 & 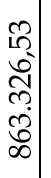 & 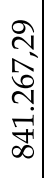 & $\begin{array}{l}\infty \\
\infty \\
- \\
\vec{\delta} \\
\stackrel{-}{0} \\
\infty\end{array}$ & 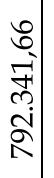 & 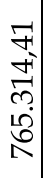 & 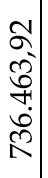 & 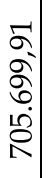 & $\begin{array}{l}\infty \\
\infty \\
\hat{N} \\
\hat{N} \\
\hat{N} \\
\hat{\sigma}\end{array}$ & 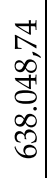 \\
\hline$\frac{\stackrel{\mathscr{J}}{\pi}}{\omega}$ & 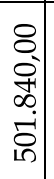 & 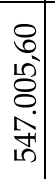 & 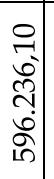 & \begin{tabular}{l} 
m. \\
$\hat{2}$ \\
$\infty$ \\
$\infty$ \\
\multirow{b}{0}{}
\end{tabular} & $\begin{array}{c}\text { ㄱ. } \\
\text { - } \\
\infty \\
m \\
\infty \\
0 \\
0\end{array}$ & 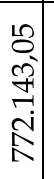 & $\begin{array}{c}\Omega \\
\hat{\sigma} \\
0 \\
\hat{ర} \\
-\overrightarrow{+} \\
\infty\end{array}$ & 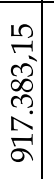 & 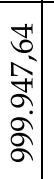 & 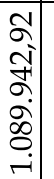 & 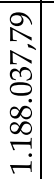 & 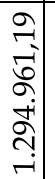 & 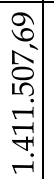 & 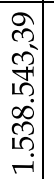 & 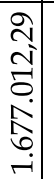 & 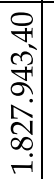 & 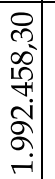 & 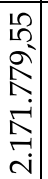 & 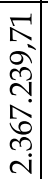 & 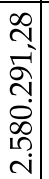 & 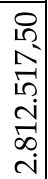 \\
\hline 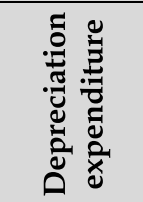 & 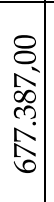 & 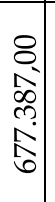 & 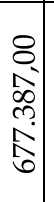 & 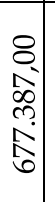 & 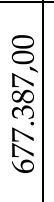 & 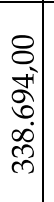 & 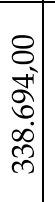 & $\begin{array}{l}8 \\
0 \\
+1 \\
\hat{0} \\
\infty \\
0 \\
0\end{array}$ & $\begin{array}{l}8 \\
0 \\
+1 \\
\hat{0} \\
\infty \\
0 \\
0\end{array}$ & $\begin{array}{l}8 \\
0 \\
+5 \\
0 \\
\infty \\
\infty \\
0 \\
0\end{array}$ & $\begin{array}{l}8 \\
0 \\
+5 \\
0 \\
\infty \\
\infty \\
0 \\
0\end{array}$ & 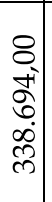 & 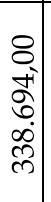 & 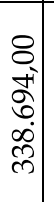 & $\begin{array}{l}8 \\
0 \\
+1 \\
\hat{0} \\
\infty \\
0 \\
0\end{array}$ & $\begin{array}{l}8 \\
8 \\
+ \\
6 \\
\infty \\
\infty \\
m \\
m\end{array}$ & $\begin{array}{l}8 \\
8 \\
+ \\
0 \\
\infty \\
\infty \\
0 \\
0\end{array}$ & 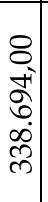 & $\begin{array}{l}8 \\
+ \\
\stackrel{+}{0} \\
\infty \\
\infty \\
m \\
m\end{array}$ & 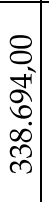 & 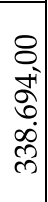 \\
\hline 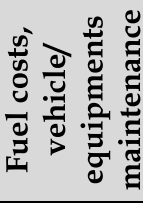 & 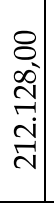 & 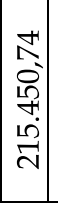 & 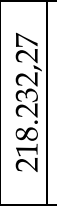 & 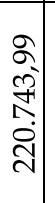 & 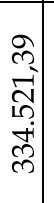 & 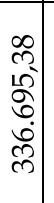 & 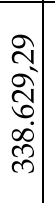 & 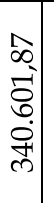 & 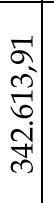 & 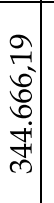 & 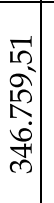 & 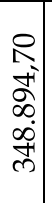 & 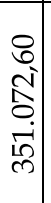 & 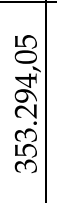 & $\begin{array}{l}\Omega \\
\Omega \\
10 \\
10 \\
10 \\
10 \\
n\end{array}$ & 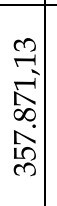 & 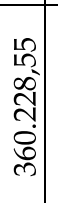 & 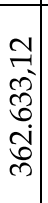 & $\begin{array}{l}\infty \\
10 \\
10 \\
0 \\
0 \\
0 \\
\infty \\
\infty\end{array}$ & 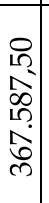 & 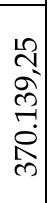 \\
\hline 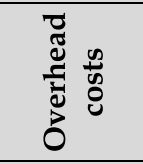 & 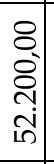 & 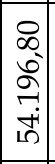 & 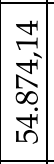 & $\begin{array}{l}10 \\
\infty \\
8 \\
8 \\
10 \\
10 \\
10\end{array}$ & 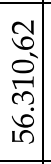 & 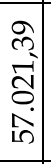 & 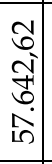 & $\begin{array}{l}\hat{~} \\
\infty \\
0 \\
0 \\
\infty \\
10\end{array}$ & $\begin{array}{l}3 \\
6 \\
\infty \\
0 \\
0 \\
0 \\
10\end{array}$ & 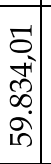 & $\begin{array}{l}9 \\
0 \\
+ \\
6 \\
8\end{array}$ & $\begin{array}{l}\infty \\
2 \\
0 \\
7 \\
\forall \\
\dot{b}\end{array}$ & $\begin{array}{l}\text { సి } \\
\text { సે } \\
\text { సู }\end{array}$ & $\begin{array}{c}0 \\
6 \\
-1 \\
8 \\
8 \\
0\end{array}$ & 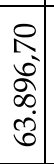 & 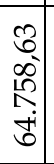 & 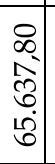 & 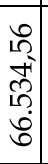 & 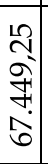 & 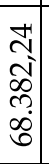 & 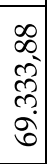 \\
\hline 幽苞 & $\begin{array}{l}5 \\
0 \\
0 \\
0 \\
0 \\
\vdots \\
=\end{array}$ & 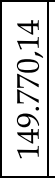 & 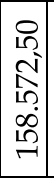 & 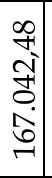 & 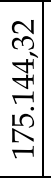 & 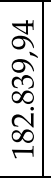 & $\begin{array}{l}-\infty \\
\infty \\
\infty \\
0 \\
8 \\
8 \\
\end{array}$ & 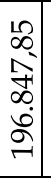 & 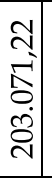 & 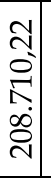 & 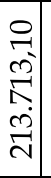 & $\begin{array}{l}0 \\
\infty \\
\Delta \\
\Delta \\
0 \\
\infty \\
\vec{N}\end{array}$ & 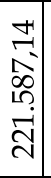 & 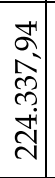 & 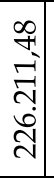 & 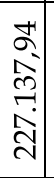 & 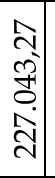 & 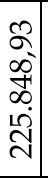 & 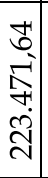 & 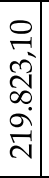 & $\begin{array}{l}\text { से } \\
\text { Dे } \\
\infty \\
+ \\
\dot{\mathbb{N}}\end{array}$ \\
\hline 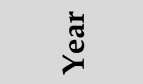 & $\begin{array}{l}\text { ஓे } \\
\text { ¿े }\end{array}$ & 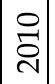 & 咅 & $\begin{array}{l}\stackrel{\sim}{ } \\
\stackrel{\sim}{\sim}\end{array}$ & $\begin{array}{c}m \\
\stackrel{\sim}{\sim}\end{array}$ & $\begin{array}{l}+ \\
\stackrel{d}{8}\end{array}$ & $\begin{array}{c}10 \\
\stackrel{2}{0} \\
\text { | }\end{array}$ & 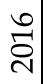 & 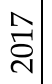 & 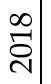 & $\begin{array}{c}\sigma \\
\bar{\delta}\end{array}$ & 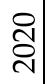 & 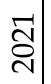 & $\begin{array}{l}\text { ָิ } \\
\text { ָิ }\end{array}$ & $\begin{array}{c}\text { సి } \\
\text { సి }\end{array}$ & 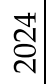 & 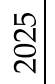 & 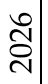 & సิ & 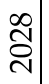 & సิ \\
\hline
\end{tabular}

Table 3. Prediction of the total expenditure of the operation of the proposed project (Euro) 
The ascertainment of the depreciation was achieved by applying depreciation rates on the input value of assets. It is included in operating expenses. Depreciation for buildings / facilities, equipment and machinery was calculated by applying depreciation rates on the value of the depreciable asset. The depreciation rates is given by:

$$
\mathrm{Ca}(\%)=\frac{100}{t}
$$

where:

Ca - depreciation rate, expressed as a percentage;

$\mathrm{t}$ - the normal duration of the asset, expressed in years.

The results for depreciation rates for construction / facilities, equipment and machinery are: $11 \%, 20 \%$ and $5 \%$.

Storage fee costs were obtained by multiplying the total quantity of stored waste by the presumed value of storage fee. In order to safely store the final waste, a storage fee is considered that is applied to each ton of deposited waste. This fee is expected to grow in order to determine the local authorities to take measures to reduce the quantities sent to storage thus encouraging their sale.

This waste management plan proposed for Comunitatea Urbană Arieş involves, in addition to costs resulting from the incineration explained above, also the operating expenses for the first waste management scheme in the area (proposed scheme from volume no. 27 E/October/2009 of the Transylvanian Review of Administrative Sciences journal).

In the integrated concepts of waste management, the incineration process can be an important component.

The reintroduction of separately collected organic waste fractions in the flow of substances, we can obtain an apparent easing of the waste deposits from the researched villages and towns, and the recoverable heat fraction is optimized in terms of its composition and its thermal power.

The purpose of incineration is:

- minimizing waste quantities;

- destroying dangerous biodegradable components;

- $\quad$ transforming waste in inert (inactive) material;

- $\quad$ recovery of energy contained in the incinerated waste.

All waste incinerators must meet the objectives of European and national legislation. In parallel, incinerators must meet the conditions regarding energy recovery from waste, i.e. heat recovery and other forms of energy derived from waste incineration. Incinerators should be operated so that the final ashes can be sorted to recover recyclable metals and to be used in construction, with no impact on soil and groundwater.

Every civilized country must implement and use the waste recovery infrastructure to protect the environment and life itself. Environmental benefits of recycling are much more 
effective than any other action to protect the environment. Recycling conserves energy. Much less energy is needed to transform recycled materials into new products, compared with the production of raw materials.

Following the implementation of such a project it is very important to consider the social and environmental benefits:

- the improvement of water, soil and air quality in the area where a waste management project has been implemented;

- $\quad$ increase of life expectancy due to limitation of pollution;

- local development generated by the project (increasing land prices, development of tourism and investor-friendliness, new employment opportunities etc.);

- $\quad$ awareness-raising among the general public as to the resources contained in household waste.

\begin{tabular}{|c|c|c|c|c|c|c|}
\hline Year & $\begin{array}{c}\text { Income from } \\
\text { electricity } \\
\text { sale }\end{array}$ & $\begin{array}{c}\text { Income from } \\
\text { green } \\
\text { certificate stock } \\
\text { exchange }\end{array}$ & $\begin{array}{c}\text { Income } \\
\text { from by- } \\
\text { products } \\
\text { recovery }\end{array}$ & $\begin{array}{c}\text { Total } \\
\text { incineration } \\
\text { income }\end{array}$ & $\begin{array}{c}\text { Generated } \\
\text { energy } \\
\text { (MWh) }\end{array}$ & $\begin{array}{c}\text { Number } \\
\text { of green } \\
\text { certificates }\end{array}$ \\
\hline 2009 & $205.857,09$ & $1.157 .946,14$ & $85.773,79$ & 1.449 .577 .01 & $8.577,38$ & $25.732,14$ \\
\hline 2010 & $187.212,68$ & $1.148 .805,06$ & $85.096,67$ & $1.421 .114,41$ & $8.509,67$ & $25.529,00$ \\
\hline 2011 & $185.563,56$ & $1.138 .685,48$ & $84.347,07$ & $1.408 .596,11$ & $8.434,71$ & $25.304,12$ \\
\hline 2012 & $183.746,73$ & $1.127 .536,74$ & $83.521,24$ & $1.394 .804,71$ & $8.352,12$ & $25.056,37$ \\
\hline 2013 & $181.753,54$ & $1.115 .305,81$ & $82.615,25$ & $1.379 .674,60$ & $8.261,52$ & $24.784,57$ \\
\hline 2014 & $179.574,94$ & $1.101 .937,12$ & $81.624,97$ & $1.363 .137,03$ & $8.162,50$ & $24.487,49$ \\
\hline 2015 & $177.201,43$ & $1.087 .372,44$ & $80.546,11$ & $1.345 .119,98$ & $8.054,61$ & $24.163,83$ \\
\hline 2016 & $174.623,09$ & $1.071 .550,78$ & $79.374,13$ & $1.325 .548,00$ & $7.937,41$ & $23.812,24$ \\
\hline 2017 & $171.829,49$ & $1.054 .408,26$ & $78.104,32$ & $1.304 .342,06$ & $7.810,43$ & $23.431,29$ \\
\hline 2018 & $168.809,74$ & $1.035 .877,94$ & $76.731,70$ & $1.281 .419,37$ & $7.673,17$ & $23.019,51$ \\
\hline 2019 & $165.552,40$ & $1.015 .889,72$ & $75.251,09$ & $1.256 .693,21$ & $7.525,11$ & $22.575,33$ \\
\hline 2020 & $162.045,51$ & $994.370,16$ & $73.657,05$ & $1.230 .072,72$ & $7.365,70$ & $22.097,11$ \\
\hline 2021 & $158.276,53$ & $971.242,35$ & $71.943,88$ & $1.201 .462,75$ & $7.194,39$ & $21.583,16$ \\
\hline 2022 & $154.232,34$ & $946.425,70$ & $70.105,61$ & $1.170 .763,65$ & $7.010,56$ & $21.031,68$ \\
\hline 2023 & $149.899,17$ & $919.835,84$ & $68.135,99$ & $1.137 .871,00$ & $6.813,60$ & $20.440,80$ \\
\hline 2024 & $145.262,64$ & $891.384,37$ & $66.028,47$ & $1.102 .675,48$ & $6.602,85$ & $19.808,54$ \\
\hline 2025 & $140.307,64$ & $860.978,71$ & $63.776,20$ & $1.065 .062,55$ & $6.377,62$ & $19.132,86$ \\
\hline 2026 & $135.018,38$ & $828.521,90$ & $61.371,99$ & $1.024 .912,28$ & $6.137,20$ & $18.411,60$ \\
\hline 2027 & $129.378,32$ & $793.912,40$ & $58.808,33$ & $982.099,04$ & $5.880,83$ & $17.642,50$ \\
\hline 2028 & $123.370,11$ & $757.043,84$ & $56.077,32$ & $936.491,27$ & $5.607,73$ & $16.823,20$ \\
\hline 2029 & $116.975,60$ & $717.804,83$ & $53.170,73$ & $887.951,16$ & $5.317,07$ & $15.951,22$ \\
\hline
\end{tabular}

Table 4. Prediction of the total income obtained from the waste incineration process (Euro) 
To determine the total revenue resulting from the operation of this waste management scheme, with the revenues from exploitation of recoverable materials (paper/cardboard, glass, plastic, metal and organic waste) and the income from the sanitation charges, revenues from the incineration process will be taken into account, as follows:

- income from sale of electricity: about 12 euros per ton of waste processed; one tonne of waste processed $=0,5 \mathrm{MW}$ recovered energy;

- $\quad$ income from the green certificate stock of exchange: cca 45 Euro per green certificate; each MWh of recovered energy yields 2 green certificates;

- income resulting the recovery of by-products resulting from incineration: about 5 euros per ton of processed waste (mineral acids, furnace slag used in construction materials industry, etc.).

\begin{tabular}{|c|c|c|c|c|c|}
\hline Year & $\begin{array}{c}\text { Income from } \\
\text { materials } \\
\text { recovery }\end{array}$ & $\begin{array}{c}\text { Population } \\
\text { service } \\
\text { charge }\end{array}$ & $\begin{array}{c}\text { Companies } \\
\text { service } \\
\text { charge }\end{array}$ & $\begin{array}{c}\text { Incineration } \\
\text { income }\end{array}$ & $\begin{array}{c}\text { TOTAL } \\
\text { INCOME }\end{array}$ \\
\hline 2009 & $964.547,68$ & $1.413 .360,00$ & $234.410,71$ & $1.449 .577,01$ & $4.061 .895,41$ \\
\hline 2010 & $1.002 .840,13$ & $1.440 .120,56$ & $239.567,75$ & $1.421 .114,41$ & $4.103 .642,85$ \\
\hline 2011 & $1.042 .866,71$ & $1.467 .387,80$ & $244.838,24$ & $1.408 .596,11$ & $4.163 .688,86$ \\
\hline 2012 & $1.084 .708,15$ & $1.495 .171,32$ & $250.224,68$ & $1.394 .804,71$ & $4.224 .908,86$ \\
\hline 2013 & $1.128 .448,95$ & $1.523 .480,90$ & $255.729,62$ & $1.379 .674,60$ & $4.287 .334,07$ \\
\hline 2014 & $1.174 .177,57$ & $1.552 .326,48$ & $261.355,68$ & $1.363 .137,03$ & $4.350 .996,75$ \\
\hline 2015 & $1.221 .986,59$ & $1.581 .718,23$ & $267.105,50$ & $1.345 .119,98$ & $4.415 .930,30$ \\
\hline 2016 & $1.271 .972,95$ & $1.611 .666,49$ & $272.981,82$ & $1.325 .548,00$ & $4.482 .169,26$ \\
\hline 2017 & $1.324 .238,11$ & $1.642 .181,78$ & $278.987,42$ & $1.304 .342,06$ & $4.549 .749,38$ \\
\hline 2018 & $1.378 .888,29$ & $1.673 .274,85$ & $285.125,15$ & $1.281 .419,37$ & $4.618 .707,66$ \\
\hline 2019 & $1.436 .034,66$ & $1.704 .956,63$ & $291.397,90$ & $1.256 .693,21$ & $4.689 .082,40$ \\
\hline 2020 & $1.495 .793,62$ & $1.737 .238,28$ & $297.808,65$ & $1.230 .072,72$ & $4.760 .913,28$ \\
\hline 2021 & $1.558 .287,01$ & $1.770 .131,15$ & $304.360,44$ & $1.201 .462,75$ & $4.834 .241,36$ \\
\hline 2022 & $1.623 .642,35$ & $1.803 .646,82$ & $311.056,37$ & $1.170 .763,65$ & $4.909 .109,19$ \\
\hline 2023 & $1.691 .993,17$ & $1.837 .797,06$ & $317.899,61$ & $1.137 .871,00$ & $4.985 .560,85$ \\
\hline 2024 & $1.763 .479,22$ & $1.872 .593,91$ & $324.893,40$ & $1.102 .675,48$ & $5.063 .642,02$ \\
\hline 2025 & $1.838 .246,80$ & $1.908 .049,61$ & $332.041,06$ & $1.065 .062,55$ & $5.143 .400,02$ \\
\hline 2026 & $1.916 .449,07$ & $1.944 .176,62$ & $339.345,96$ & $1.024 .912,28$ & $5.224 .883,94$ \\
\hline 2027 & $1.998 .246,35$ & $1.980 .987,66$ & $346.811,57$ & $982.099,04$ & $5.308 .144,62$ \\
\hline 2028 & $2.083 .806,46$ & $2.018 .495,68$ & $354.441,43$ & $936.491,27$ & $5.393 .234,83$ \\
\hline 2029 & $2.173 .305,09$ & $2.056 .713,88$ & $362.239,14$ & $887.951,16$ & $5.480 .209,27$ \\
\hline
\end{tabular}

Table 5. Prediction of the total income obtained from the waste management scheme proposed for CUA (Euro) 
For the incineration process to be economically efficient and to qualify for additional green certificates from non-conventional energy recovery, it's necessary to combine at least two forms of energy recovery, which usually means combining electricity generation from a steam turbine coupled with at least a household heating system or providing technological steam. Electricity is traded separately from green certificates.

\section{Research results}

The analysis of the efficiency for an investment project is performed using an instrument of investigation which is represented by the investment efficiency indicators. Using these indicators for measuring the efficiency of investments focuses on a very important aspect: the investments' efficiency can be expressed using a single indicator or multiple indicators. Both in our country's economic practice and the experience of other countries show the usage of a wider or a smaller range of indicators, but never a single indicator. (Bințințan, 2005)

The time factor has a major influence on the project's performance. The operation through which a certain amount, regardless of its nature, be it income or expenditure, shall be recalculated in monetary units of a reference moment in time, is called an update.

In fact it is a recalculation of that amount and not a simple translation of it from year to year. The update's base is the annual rate of fruition of a monetary unit, which is symbolized by "a" and it is called the discount rate.

This scheme's efficiency was proven by using two efficiency determination criteria, which are very often used in the area:

a. Dynamic criteria or basic on updating, in which we determined the following investment efficiency indicators: (Bințințan, 2004)

- the benefit-cost ratio BCR:

By its content, the benefit-costs ratio indicates the gain, the return or the reward for invested capital, in the form of an absolute value. This indicator summarizes the investment efforts and effects for the entire lifetime of the project; it reflects the difference between the total updated income and the total updated expenditures (including investment costs).

- the net present value NPV:

This indicator also refers to the effort and the effects of the investment project for the whole duration of the project; it reflects the difference between the present value of benefits and the present value of costs (capital expenditure and operating costs).

- the internal rate of return IRR represents the fundamental indicator for accepting a project. It refers to that updating rate for which the present value of the benefits equals the present value of the costs, so the benefit-cost ratio equals 1 and NPV equals 0 . The calculation of the internal rate of return IRR is carried out in a succession of approximations, i.e. the net present value is determined for an appropriate discount rate, which is considered to be the minimum rate, for which the NPV must be positive. Then, the NPV is calculated for a rate superior to the minimum rate that is high enough to obtain a negative NPV. 
These indicators have been explained in detail in the first project proposed for the investigated area, presented in volume no. 27E/Octombrie/2009. (Scorțar et al., 2009)

b. Double rate criteria or integrated criteria: wherein the following efficiency indicators have been determined: (Todea, 2008)

- the integrated internal rate of return (IIRR)

- $\quad$ the integrated net present value (INPV).

The integrated internal rate of return (IIRR) involves the reinvestment of net treasury flows at a reinvestment rate different from the internal profitability index.

Compared to the internal rate of return (IRR), a first advantage of this indicator would be the fact that it allows for a more accurate comparison of two competing investment project against a unique net treasury flows reinvestment ratio.

A second advantage consists in the fact that that IIRR enables comparisons between investment projects of different durations (lifetimes).

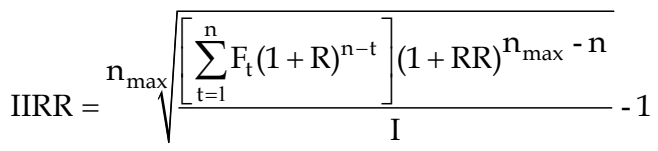

where:

I - capital expenditures updated on a specific updating rate;

$\mathrm{n}$ - the project duration for which IRR is determined;

$F_{t}$ - the net flow of treasury for the year $t$, that may be positive or negative;

$\mathrm{RR}$ - net treasury flows reinvestment ratio;

$\mathrm{R}$ - updating rate;

$\mathrm{n}_{\max }$ - the longest lifetime of the projects to be compared

$\operatorname{IIRR}=8,03 \%$

The computed value of the integrated internal rate of return justifies a capital investment in this waste management scheme.

This value has been achieved by reinvesting the treasury flows at a $10 \%$ reinvestment rate that exceeds the $5 \%$ updating rate.

The implicit hypothesis of net treasury flows reinvestment during the implementation period of the intended investment target at a higher reinvestment rate that the updating rate $R$ will be highlighted by starting form the classical net present value (NPV) calculation formula that generates a general integrated net present value (INPV) calculation relation:

$$
\mathrm{INPV}=\frac{\left[\sum_{\mathrm{t}=1}^{\mathrm{n}} \mathrm{F}_{\mathrm{t}}(1+\mathrm{R})^{\mathrm{n}-\mathrm{t}}\right](1+R R)^{\mathrm{n}_{\max }-\mathrm{n}}}{(1+\mathrm{k})^{\mathrm{n} \max }}-\mathrm{I}
$$

where: 
I - updated amount based on a $\mathrm{k}$ updating rate of the capital expenditures sustained during the entire project lifetime;

$\mathrm{n}$ - the project duration for which IRR is determined;

$F_{t}$ - the net flow of treasury for the year $t$, that may be positive or negative;

$\mathrm{RR}$ - net treasury flows reinvestment rate;

$\mathrm{R}$ - rate that may be a reinvestment rate if the net treasury flows are positive or negative;

$\mathrm{k}$ - financing costs, if the net treasury flows are negative;

$\mathrm{n}_{\max }$ - the longest lifetime of the projects to be compared.

$\mathrm{INPV}=12.998 .737$ euro

This amount represents the income gathered by the end of the 21 years - lifetime of the proposed waste management scheme that is achieved by reinvesting the treasury flows at a $10 \%$ - reinvestment rate that exceeds the $5 \%$ - capital expenditure updating rate.

The income realized at the end of the 21 years - period is significant, considering the investment start-up expenditures amounting to a total of 14.629.636 Euro, demonstrating the viability of the proposed project.

\begin{tabular}{|c|c|c|c|c|c|c|c|}
\hline Year & $\begin{array}{c}\text { Up-dating } \\
\text { factor } \\
\mathbf{( 5 \% )}\end{array}$ & $\begin{array}{c}\text { Total } \\
\text { income }\end{array}$ & $\begin{array}{c}\text { Total } \\
\text { updated } \\
\text { income }\end{array}$ & $\begin{array}{c}\text { Total } \\
\text { operation } \\
\text { expenditure }\end{array}$ & $\begin{array}{c}\text { Updated } \\
\text { operation } \\
\text { expenditure }\end{array}$ & $\begin{array}{c}\text { Invest- } \\
\text { ment } \\
\text { value }\end{array}$ & $\begin{array}{c}\text { Updated } \\
\text { investment } \\
\text { value }\end{array}$ \\
\hline 2008 & 0,952 & - & - & - & - & 8.000 .000 & $7.619 .047,6$ \\
\hline 2009 & 0,907 & $4.061 .895,4$ & $3.684 .258,88$ & $3.017 .940,81$ & $2.737 .361,2$ & 6.629 .636 & $6.013 .275,3$ \\
\hline 2010 & 0,864 & $4.103 .642,8$ & $3.544 .880,98$ & $3.065 .955,84$ & $2.648 .487,9$ & - & - \\
\hline 2011 & 0,823 & $4.163 .688,8$ & $3.425 .477,13$ & $3.115 .073,92$ & $2.562 .779,0$ & - & - \\
\hline 2012 & 0,784 & $4.224 .908,8$ & $3.310 .326,65$ & $3.167 .066,69$ & $2.481 .479,6$ & - & - \\
\hline 2013 & 0,746 & $4.287 .334,0$ & $3.199 .274,69$ & $3.333 .591,22$ & $2.487 .577,0$ & - & - \\
\hline 2014 & 0,711 & $4.350 .996,7$ & $3.092 .172,16$ & $3.053 .404,73$ & $2.169 .997,7$ & - & - \\
\hline 2015 & 0,677 & $4.415 .930,3$ & $2.988 .875,45$ & $3.115 .398,10$ & $2.108 .624,0$ & - & - \\
\hline 2016 & 0,645 & $4.482 .169,2$ & $2.889 .246,27$ & $3.181 .988,53$ & $2.051 .138,1$ & - & - \\
\hline 2017 & 0,614 & $4.549 .749,3$ & $2.793 .151,44$ & $3.253 .424,90$ & $1.997 .320,6$ & - & - \\
\hline 2018 & 0,585 & $4.618 .707,6$ & $2.700 .462,71$ & $3.330 .134,60$ & $1.947 .060,7$ & - & - \\
\hline 2019 & 0,557 & $4.689 .082,4$ & $2.611 .056,54$ & $3.412 .653,49$ & $1.900 .293,1$ & - & - \\
\hline 2020 & 0,530 & $4.760 .913,2$ & $2.524 .813,96$ & $3.501 .570,53$ & $1.856 .957,6$ & - & - \\
\hline 2021 & 0,505 & $4.834 .241,3$ & $2.441 .620,39$ & $3.597 .532,80$ & $1.816 .998,5$ & - & - \\
\hline 2022 & 0,481 & $4.909 .109,1$ & $2.361 .365,46$ & $3.701 .251,01$ & $1.780 .365,0$ & - & - \\
\hline 2023 & 0,458 & $4.985 .560,8$ & $2.283 .942,87$ & $3.813 .505,59$ & $1.747 .010,8$ & - & - \\
\hline 2024 & 0,436 & $5.063 .642,0$ & $2.209 .250,24$ & $3.935 .153,24$ & $1.716 .894,3$ & - & - \\
\hline 2025 & 0,416 & $5.143 .400,0$ & $2.137 .188,95$ & $4.067 .134,10$ & $1.689 .978,2$ & - & - \\
\hline 2026 & 0,396 & $5.224 .883,9$ & $2.067 .663,99$ & $4.210 .479,68$ & $1.666 .229,7$ & - & - \\
\hline 2027 & 0,377 & $5.308 .144,6$ & $2.000 .583,88$ & $4.366 .321,41$ & $1.645 .620,6$ & - & - \\
\hline 2028 & 0,359 & $5.393 .234,8$ & $1.935 .860,46$ & $4.535 .900,03$ & $1.628 .126,6$ & - & - \\
\hline 2029 & 0,342 & $5.480 .209,2$ & $1.873 .408,83$ & $4.720 .575,85$ & $1.613 .728,2$ & - & - \\
\hline & Total & $56.074 .881,9$ & & $42.254 .029,3$ & & $\mathbf{1 3 . 6 3 2 . 3 2 2 , 9}$ \\
\hline & & Updating factor: 0,05 & & $N P V(e u r o):$ & $\mathbf{1 8 8 . 5 2 9 , 6 5}$ & \\
\hline & & & & & & \\
\hline
\end{tabular}

Table 6. The structure of the proposed investment project (euro) 
In the presented scheme, the year 2008 was considered the start of the construction for the investment objective, the capital investment is phased as follows: in the first, when the construction started, the initial investment was 8 million euro, and in the next year the difference of 6.629.636 euro was invested. This project was designed to come into operation in 2009, bringing income as a result of operating the facility. As shown in Table 6, total income and total expenditure have been updated to a $5 \%$ updating rate (the rate recommended for environmental projects) and were thus obtained updating income and updating expenditure, required to determine the efficiency indicators of this project.

In the waste management scheme proposed for Comunitatea Urbană Arieş we took into account the pre-existing endowments of the local sanitation systems and this is why we also considered the fact that part of the population living in private houses and part of the economic agents already own adequate containers for waste collection.

In this respect, we included investment cots just for the difference in the containers inventory, costs which would be directly recovered from the direct beneficiaries based on a conscription tariff.

\begin{tabular}{|c|c|c|c|c|c|c|}
\hline Year & $\begin{array}{c}\text { Invest- } \\
\text { ment } \\
\text { value } \\
\text { (in euro) }\end{array}$ & $\begin{array}{c}\text { Operation } \\
\text { expenditures }\end{array}$ & $\begin{array}{c}\text { Annual } \\
\text { income } \\
\text { (euro) }\end{array}$ & $\begin{array}{c}\text { Cashflow } \\
\text { (euro) }\end{array}$ & $\begin{array}{c}\text { Updating } \\
\text { factor } \\
\mathbf{a}=\mathbf{5} \% \mathbf{)}\end{array}$ & $\begin{array}{c}\text { Updated } \\
\text { cash flow }\end{array}$ \\
\hline 2008 & 8.000 .000 & 0,00 & 0,00 & $-8.000 .000,00$ & 0,951 & $-7.607 .006,81$ \\
\hline 2009 & 6.629 .636 & $3.017 .940,81$ & $4.061 .895,41$ & $-5.585 .681,40$ & 0,904 & $-5.050 .376,98$ \\
\hline 2010 & 0,00 & $3.065 .955,84$ & $4.103 .642,85$ & $1.037 .687,01$ & 0,860 & $892.149,91$ \\
\hline 2011 & 0,00 & $3.115 .073,92$ & $4.163 .688,86$ & $1.048 .614,94$ & 0,818 & $857.257,54$ \\
\hline 2012 & 0,00 & $3.167 .066,69$ & $4.224 .908,86$ & $1.057 .842,17$ & 0,777 & $822.318,33$ \\
\hline 2013 & 0,00 & $3.333 .591,22$ & $4.287 .334,07$ & $953.742,85$ & 0,739 & $704.975,79$ \\
\hline 2014 & 0,00 & $3.053 .404,73$ & $4.350 .996,75$ & $1.297 .592,02$ & 0,703 & $912.021,12$ \\
\hline 2015 & 0,00 & $3.115 .398,10$ & $4.415 .930,30$ & $1.300 .532,20$ & 0,668 & $869.183,86$ \\
\hline 2016 & 0,00 & $3.181 .988,53$ & $4.482 .169,26$ & $1.300 .180,73$ & 0,635 & $826.262,59$ \\
\hline 2017 & 0,00 & $3.253 .424,90$ & $4.549 .749,38$ & $1.296 .324,48$ & 0,604 & $783.342,89$ \\
\hline 2018 & 0,00 & $3.330 .134,60$ & $4.618 .707,66$ & $1.288 .573,06$ & 0,575 & $740.407,91$ \\
\hline 2019 & 0,00 & $3.412 .653,49$ & $4.689 .082,40$ & $1.276 .428,91$ & 0,546 & $697.400,82$ \\
\hline 2020 & 0,00 & $3.501 .570,53$ & $4.760 .913,28$ & $1.259 .342,74$ & 0,520 & $654.264,84$ \\
\hline 2021 & 0,00 & $3.597 .532,80$ & $4.834 .241,36$ & $1.236 .708,56$ & 0,494 & $610.943,18$ \\
\hline 2022 & 0,00 & $3.701 .251,01$ & $4.909 .109,19$ & $1.207 .858,18$ & 0,470 & $567.378,95$ \\
\hline 2023 & 0,00 & $3.813 .505,59$ & $4.985 .560,85$ & $1.172 .055,26$ & 0,447 & $523.515,06$ \\
\hline 2024 & 0,00 & $3.935 .153,24$ & $5.063 .642,02$ & $1.128 .488,78$ & 0,425 & $479.294,18$ \\
\hline 2025 & 0,00 & $4.067 .134,10$ & $5.143 .400,02$ & $1.076 .265,93$ & 0,404 & $434.658,64$ \\
\hline 2026 & 0,00 & $4.210 .479,68$ & $5.224 .883,94$ & $1.014 .404,26$ & 0,384 & $389.550,36$ \\
\hline 2027 & 0,00 & $4.366 .321,41$ & $5.308 .144,62$ & $941.823,22$ & 0,365 & $343.910,75$ \\
\hline 2028 & 0,00 & $4.535 .900,03$ & $5.393 .234,83$ & $857.334,80$ & 0,347 & $297.680,67$ \\
\hline 2029 & 0,00 & $4.720 .575,85$ & $5.480 .209,27$ & $759.633,42$ & 0,330 & $250.800,31$ \\
\hline Total & & & & & & -66 \\
\hline
\end{tabular}

Table 7. Determination of the internal rate of return (euro) 
The internal rate of return IRR calculation is done through successive approximations, where I determine the net present value at a corresponding updating rate, considered to be the minimum rate and for which it should be positive. Then, I calculate the net present value at a higher updating rate than the minimum rate, high enough to obtain a negative net present value. In the end, the exact determination of the internal financial rate of return is done through interpolation, according to the relation:

$$
\mathrm{IRR}=\mathrm{R}_{\text {min }}+\left(\mathrm{R}_{\text {max }}-\mathrm{R}_{\text {min }}\right) \times \frac{\mathrm{NPV}(+)}{\mathrm{NPV}(+)+|\mathrm{NPV}(-)|}
$$

where:

IRR - the internal financial rate of return;

$\mathrm{R}_{\min }$ - the minimum present rate;

$\mathrm{R}_{\max }$ - the maximum present rate;

NPV (+) - the positive net present value, obtained at the minimum rate;

NPV (-) - the negative net updated income, obtained at maximum rate.

IRR $=5,17 \%$

The internal rate of return is higher than the minimum rate $(5 \%)$, which proves the viability of the proposed project.

Graphically, the internal financial rate of return is at the intersection of the abscissa with the line that joins the points of the coordinates minimum rate and positive net present value, respectively maximum rate and negative net present value.

The higher the internal rate of return, the more viable the project is, its efficiency being increased. The efficiency condition of a project from the point of view of this indicator is that IRR should be superior to the corresponding updating rate.

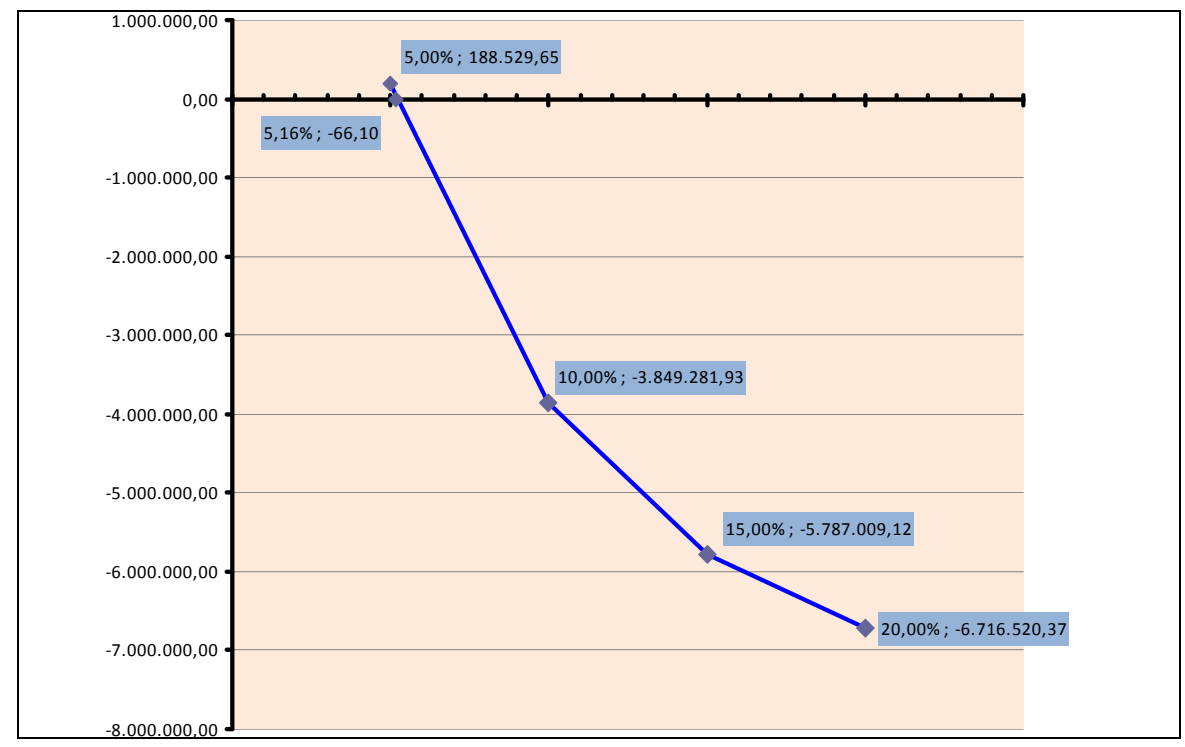

Fig. 1. Graphical illustration of the internal rate of return 
The graph illustrates the net present value, determined upon several updating rates $(5 \%$; $5,16 \% ; 10 \% ; 15 \% ; 20 \%$ ) by means of Microsoft Excel.

The net present values calculated on updating rates smaller than 5,15\%; the profitability of this project is therefore close to the minimum (5\% is considered to be the lowest rate of profitability for the proposed project).

By applying the same calculation method of the financial efficiency indicators determined for the first waste management scheme, the results are:

$\mathrm{NPV}=188.529,65$ euro

$\mathrm{BCR}=1,01$

IRR $=5,17 \%$

$\operatorname{IIRR}=8,03 \%$

$\mathrm{INPV}=12.998 .737$ euro

By applying the Microsoft Excel calculation software, the resulting integrated internal rate of return for the proposed project is $8,03 \%$ in case of financial analysis, which justify the profitability of the project for the area under scrutiny.

The feasibility of the project also results from the determined updated integrated net present values (12.998.737 euro).

The analysis of determined results demonstrates the overall feasibility of the project, though not without a few reservations:

- the initial project start-up investment is extremely high (14.629.636 euro), but the income amounts cover the expenditures required to keep the scheme in operation;

- from the point of view of the dynamic criteria used in financial analysis (net present value, benefit-cost ratio, internal rate of return), this project is very close to the bottom line of profitability, with the values of these indicators being close to $5 \%$;

- the income gained after 21 years of operation, as determined by financial analysis, is not significant (188.529,65 euro), i.e. a financial profitability of 5,17\%;

- $\quad$ on the other hand, the values calculated for double rate indicators (integrated internal rate of return, integrated net present value), that are considered to be relevant in the economic practice, prove the profitability of this project against a unique net treasury flow reinvestment ratio $(10 \%)$.

In order to finish the financial efficiency of the project proposed to Comunitatea Urbană Arieş Cluj county a detailed analysis of internal rate of return sensitivity, by studying the financial indicators' trends according to the evolution of the variables with the greatest impact on the project's profitability.

The sensitivity of this project to the changing of the same relevant variables considered for the first project has been determined by performing the project's internal rate of return sensitivity analysis. To this purpose, the relevant variables have been modified within +/$20 \%$ and the internal rate of return has been recalculated under the new circumstances. (Bințințan, 2004).

The selected variables are those whose effects on economic feasibility and financial durability are considered significant. 
Critical parameters: SERVICE PERFORMANCE FEE

\begin{tabular}{|l|l|l|l|l|l|l|l|l|l|}
\hline $\mathbf{5 , 1 7} \%$ & $\mathbf{0 , 8 0}$ & $\mathbf{0 , 8 5}$ & $\mathbf{0 , 9 0}$ & $\mathbf{0 , 9 5}$ & $\mathbf{1 , 0 0}$ & $\mathbf{1 , 0 5}$ & $\mathbf{1 , 1 0}$ & $\mathbf{1 , 1 5}$ & $\mathbf{1 , 2 0}$ \\
\hline & $-2,04 \%$ & $0,62 \%$ & $2,5 \%$ & $4,02 \%$ & $\mathbf{5 , 1 7} \%$ & $6,09 \%$ & $6,86 \%$ & $7,50 \%$ & $8,04 \%$ \\
\hline
\end{tabular}

Critical parameters: SALE PRICE OF RECYCLABLE MATERIALS

\begin{tabular}{|l|l|l|l|l|l|l|l|l|l|}
\hline $\mathbf{5 , 1 7} \%$ & $\mathbf{0 , 8 0}$ & $\mathbf{0 , 8 5}$ & $\mathbf{0 , 9 0}$ & $\mathbf{0 , 9 5}$ & $\mathbf{1 , 0 0}$ & $\mathbf{1 , 0 5}$ & $\mathbf{1 , 1 0}$ & $\mathbf{1 , 1 5}$ & $\mathbf{1 , 2 0}$ \\
\hline & $-7,9 \%$ & $-2,2 \%$ & $1,24 \%$ & $3,53 \%$ & $\mathbf{5 , 1 7} \%$ & $6,39 \%$ & $7,35 \%$ & $8,11 \%$ & $8,73 \%$ \\
\hline
\end{tabular}

Critical parameters: OPERATIONAL EXPENDITURES

\begin{tabular}{|l|l|l|l|l|l|l|l|l|l|}
\hline $\mathbf{5 , 1 7} \%$ & $\mathbf{0 , 8 0}$ & $\mathbf{0 , 8 5}$ & $\mathbf{0 , 9 0}$ & $\mathbf{0 , 9 5}$ & $\mathbf{1 , 0 0}$ & $\mathbf{1 , 0 5}$ & $\mathbf{1 , 1 0}$ & $\mathbf{1 , 1 5}$ & $\mathbf{1 , 2 0}$ \\
\hline & $9,3 \%$ & $8,65 \%$ & $7,81 \%$ & $6,7 \%$ & $\mathbf{5 , 1 7} \%$ & $2,9 \%$ & $-0,78 \%$ & $-7,81 \%$ & $-8,5 \%$ \\
\hline
\end{tabular}

Critical parameters: COMBINED IMPACT (OF THE THREE CRITICAL PARAMETERS)

\begin{tabular}{|l|l|l|l|l|l|l|l|l|l|}
\hline $\mathbf{5 , 1 7} \%$ & $\mathbf{0 , 8 0}$ & $\mathbf{0 , 8 5}$ & $\mathbf{0 , 9 0}$ & $\mathbf{0 , 9 5}$ & $\mathbf{1 , 0 0}$ & $\mathbf{1 , 0 5}$ & $\mathbf{1 , 1 0}$ & $\mathbf{1 , 1 5}$ & $\mathbf{1 , 2 0}$ \\
\hline & $2,04 \%$ & $2,99 \%$ & $3,81 \%$ & $4,53 \%$ & $\mathbf{5 , 1 7} \%$ & $5,73 \%$ & $6,24 \%$ & $6,70 \%$ & $7,11 \%$ \\
\hline
\end{tabular}

Table 8. The impact of critical parameters on the internal rate of return

The variables with the greatest impact on the feasibility on the project's feasibility are: (Scorțar, 2010)

a. The variation of sanitation fees for waste materials;

b. The sale price variation of compost and recyclable materials sold to recycling facilities;

c. The variation of operation costs.

The change of the variables within $+/-20 \%$ has yielded the following results:

- the change triggers the increase of both incomes collected from sanitation services, as well as incomes collected from recovered recyclable materials, which results in the overall increase of the project's profitability;

- the change of service fees and sale price of recoverable materials within $(-20 \%,-5 \%)$ renders the project inefficient;

- the increase of project-related operational expenditures causes the internal project profitability to decrease as a result of the increasing total expenditures required by the operation of the proposed scheme; a change within $(+5 \%,+20 \%)$ renders the project inefficient;

- the combined impact of the three critical analyzed parameters on the project's internal rate of return confirms the fact that the project is sensitive to the change of the variables within $(-20 \%,-5 \%)$, which puts the feasibility of the project at risk.

\section{Conclusions}

Due to the particular features of the analyzed area (distances, population density and behavioral habits of the population) and to the fact that most of the waste materials are biodegradable and processed in the composting facility, this project does not involve a sufficient amount of incinerated waste to make it preferable over the first scheme proposed in volume no. $27 \mathrm{E} /$ October/2009; as the project proposed in this paper has a 8,03\% profitability.

The sensitivity of this project has been proven by means of sensitivity analysis, showing that a change of several critical parameters within a certain range makes the efficiency of the project vulnerable. 
The benefits that the scheme with incineration may yield are in this case overweighted by both investment expenditures almost twice as high as those required by the scheme without incineration, as well as higher operational expenditures required for providing a temperature that enables complete cremation.

The scheme that includes incineration may have a higher profitability if the service fees would increase, thus quantifying and reflecting the financing effort and also the benefits related to the longer operation of the final deposit.

Furthermore, the analysis has revealed that the efficiency of the project proposed for this area is inconceivable outside a functional recovery system for useful materials extracted from household waste (paper/cardboard, glass, metal, plastics, including organic waste and incineration products).

It is our conviction that, in addition to the responsibilities of the public authorities related to the implementation of the integrated waste management system, the general population also bears the responsibility for the success or failure of such projects. The encouragement of cooperation and communication between citizens and authorities is a crucial part of an effective sustainable waste management program.

In this context, public authorities are responsible for organizing the collection, transport and waste disposal, they are obliged to take measures such as:

- provision of waste collection system, transport, neutralization, recovery, incineration and final disposal;

- waste-collection points with a sufficient number of containers for selective collection;

- Separate collection and timely transportation of all quantities of waste produced in localities;

- Arranging for final storage of waste (landfill), appropriately sized and equipped to protect the health and the environment;

- Prohibition of waste disposal in places other than those for fixed deposits as specified by local authorities;

- Development of guidelines for businesses, institutions and individuals on how to organize waste management;

- Association with other local authorities and contracted cooperation with businesses, in order to achieve public waste management projects;

- Organization of actions on environmental restoration.

In Romania, the current research focuses on waste management basis for strategies, action plans, strategic planning of urban waste management systems. Romanian research in the field of waste makes progress gradually, supporting those actions of the authorities to adopt waste management systems applied in the EU.

Thus, the Ministry of Environment operates two national research institutes in Bucharest (Institutul Național de Cercetare-Dezvoltare pentru Protecția Mediului - ICIM Bucuresti and Institutul Național de Cercetare-Dezvoltare pentru Ecologie Industrială - ECOIND) including laboratories and workshops specifically dedicated to research into waste management technologies.

The role of these research institutions is a scientific foundation based on national statistics and national reference databases, adopted local government strategies, and monitoring the 
implementation of commitments assumed with the signing of the Accession Treaty of Romania to the European Union (through monitoring plans).

But local research themes have been launched for other market players as well, which are quite numerous; these are represented by small business of consultancy and research, by academic structures, local institutions etc. These research topics' role was to either base regulatory decisions for local waste management facilities (environmental impact studies) and local strategies for sustainable development, or to show what happens after the closure of landfills (post-closure monitoring).

Developing this paper and the research results can be a starting point for the official structures of government, but also for some structures with an informational role (institutions, associations, EU bodies).

We believe that the first step to successful implementation of a sustainable waste management project is represented by a change in education, culture and mentality, so that waste should be viewed primarily as a resource and only ultimately as something to be removed.

\section{Acknowledgment}

I would like to express my gratitude to all those who gave me the possibility to complete this paper.

I would like to express my thanks to Comunitatea Urbană Arieş, Consiliul Județean Cluj, Agenția pentru Protecția Mediului Cluj, RADP Turda, S.C. SLCIAS S.A. Câmpia Turzii for allowing me to access their statistical data regarding their specific activities, programmes and actions taken during the household waste management activities.

\section{References}

Bințințan, P. (2005). Strategii şi politici de investiții. Casa Cărții de Ştiință, ISBN 973-686-798-6, Cluj-Napoca, Romania.

Bințințan, P. (2004). Managementul investiților. Aplicații. Evaluarea proiectelor. Presa Universitară Clujeană, ISBN 973-610-334-X, Cluj-Napoca, Romania.

Comunitatea Urbană Arieş, Statutul Asociației, Turda Town Hall, 2005

McDougall, F.; White, P.; Franke, M. \& Hindle, P. (2001). Integrated Solid Waste Management: a Life Cycle Inventory (2nd Edition). Blackwell Publishing, ISBN 0-632-05889-7, Great Britain

Planul Județean privind Gestionarea Deşeurilor, Consiliul Județean Cluj, Available from http://www.cjcluj.ro/UserUploadedFiles/File/programe/pjgd\%20Cluj\%20alterna tiva\%20finala.pdf

Scorțar, L. M.; Lazăr, I.; Popa, M.; Zagan Zelter, D. \& Pop, I. (2009). The financial analysis of a modern scheme for managing waste proposed for the Urban Community Aries, Cluj County. Transylvanian Review of Administrative Sciences, 27E/2009, pp.220-234, ISSN 1842-2845

Scorțar, L., M. (2010). Managementul deşeurilor menajere. Editura Alma Mater, ISBN, ClujNapoca, Romania

Todea, A. (2008). Investiții. Editura Casa Cărții de Ştiință, Cluj-Napoca, Romania. 


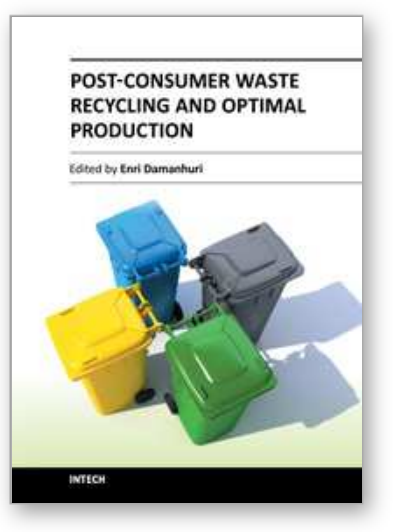

\author{
Post-Consumer Waste Recycling and Optimal Production \\ Edited by Prof. Enri Damanhuri
}

ISBN 978-953-51-0632-6

Hard cover, 294 pages

Publisher InTech

Published online 23, May, 2012

Published in print edition May, 2012

This book deals with several aspects of waste material recycling. It is divided into three sections. The first section explains the roles of stakeholders, both informal and formal sectors, in post-consumer waste activities. It also discusses waste collection programs for recycling. The second section discusses the analysis tools for recycling system. The third section focuses on the recycling process and optimal production. I hope that this book will convey both the need and means for recycling and resource conservation activities to a wide readership, at both academician and professional level, and contribute to the creation of a sound materialcycle society.

\title{
How to reference
}

In order to correctly reference this scholarly work, feel free to copy and paste the following:

Scorțar Lucia-Monica (2012). Assessing the Efficiency of a Proposed Project in Waste Management, PostConsumer Waste Recycling and Optimal Production, Prof. Enri Damanhuri (Ed.), ISBN: 978-953-51-0632-6, InTech, Available from: http://www.intechopen.com/books/post-consumer-waste-recycling-and-optimalproduction/asssessing-the-efficiency-of-a-proposed-project-in-waste-management

\section{INTECH}

open science | open minds

\section{InTech Europe}

University Campus STeP Ri Slavka Krautzeka 83/A 51000 Rijeka, Croatia Phone: +385 (51) 770447

Fax: +385 (51) 686166 www.intechopen.com

\section{InTech China}

Unit 405, Office Block, Hotel Equatorial Shanghai No.65, Yan An Road (West), Shanghai, 200040, China 中国上海市延安西路65号上海国际贵都大饭店办公楼 405 单元 Phone: +86-21-62489820

Fax: +86-21-62489821 
(C) 2012 The Author(s). Licensee IntechOpen. This is an open access article distributed under the terms of the Creative Commons Attribution 3.0 License, which permits unrestricted use, distribution, and reproduction in any medium, provided the original work is properly cited. 